\title{
Research on Modeling and Simulation of Electro Backup Hydraulic Actuation System
}

\author{
Pei Lei, Hongjun Pang, Sa Liu \\ Qing'an Group Co., Ltd, Xi'an, Shaanxi, 710077, China
}

\begin{abstract}
As one form in power-by-wire actuation system, the Electro backup hydraulic actuation system (EBHAs) has broad application prospects in the aviation field. In this paper, a numerical simulation model of the system performance for EBHAs is established. Based on the detailed description of its working principle, the mathematical models of EBHAs and its subsystems, as well as numerical model in the Matlab / Simulink environment are established, and a larger number of numerical simulations are carried out, which contain the normal working mode and the backup working mode. Furthermore, numerical simulation results are compared and analyzed with those results of experimental, which further verified the system simulation model established in this paper. It can be concluded from the results that the dynamic response of the system can meet the requirements of the design, no matter the normal or backup working modes. And the simulation results of the system sweep frequency are basically consistent with the test results, and the error is less than $10 \%$. It is clear that the simulation model presented in this paper can accurately describe the system characteristics of EBHAs, which is useful to the design of EBHAs system.
\end{abstract}

\section{Introduction}

The high performance of modern aircraft makes the aircraft develop towards high mobility, super-high speed and high power ${ }^{[1]}$, which puts forward higher requirements for the flight control actuation system. Because of the high failure rate, low efficiency, complex hydraulic pipeline and inconvenient maintenance of the electro-hydraulic servo valve, the application demand of the traditional hydraulic actuation system is gradually decreasing, and it is replaced by the power-by-wire(PBW) actuation system ${ }^{[2,3]}$. The power-by-wire actuation system no longer needs the centralized hydraulic system to supply pressure, its internal power is completed by the electric energy transmission through the electric wire ${ }^{[3]}$, which can effectively improve the reliability, survivability, maintainability and efficiency of the aircraft ${ }^{[4]}$, and has broad application prospects in both military and civil aircraft.

According to the different working principles, powerby-wire actuation system mainly includes three forms, Electro Hydro-static Actuation system (EHAs), Electro Mechanical Actuation system (EMAs) and Electro Backup Hydraulic Actuation system (EBHAs) ${ }^{[5]}$. Among them, the EBHAs can not only use the traditional electrohydraulic servo valve to drive the actuator output power, but also use the motor pump, so it inherits all the advantages of the Electro Hydraulic Servo Actuation system(EHSAs) and the EHAs ${ }^{[6]}$. But just because of this, there are many components in the EBHAs, the weight of a single actuation system is larger, the basic reliability is less than other forms of actuation system, and the control mode is complex. However, because its mission reliability has been greatly improved, which can improve the survival and safety of aircraft, it is often applied to the surface with large space of aircraft. At present, it has been successfully applied to the rudder and spoiler of A380 aircraft and the spoiler of A350 aircraft in foreign countries. However, it is in the stage of prototype development in China, and there is no engineering application example.

This paper takes the EBHAs as the research object, expounds its composition and working principle, establishes the mathematical model of each component and the whole system on this basis, simulates the whole system in Matlab / Simulink, the result shows that the EBHAs model is correct and reasonable, can provide the basis and support for the design of EBHAs, and has important significance for the research of EBHAs.

\section{Working principle of EBHAs}

The composition of EBHAs is shown in Figure 1, which is composed of controller and actuator. The actuator mainly includes electro hydraulic servo valve, motor driver, motor, hydraulic pump, hydraulic cylinder, accumulator and function conversion valve.

In general, EBHAs can be considered as a dual redundancy configuration system based on the traditional EHSAs combined with the EHAs as backup ${ }^{[7]}$. Therefore, EBHAs has two independent energy sources, the traditional centralized hydraulic source and the power required by the EHA. Any energy source can 
independently drive the common hydraulic cylinder movement through the mode conversion function. According to the structural characteristics of EBHAs, the system has two working modes, normal working mode and backup working mode. When all the electrical redundancy faults or hydraulic failures in normal working mode, the system will turn to backup working mode.

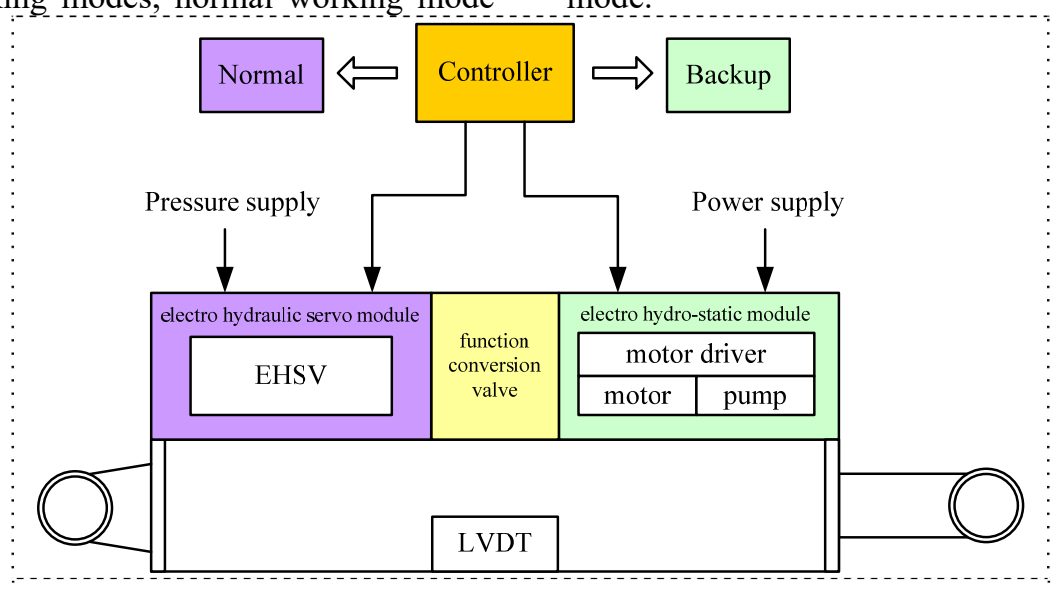

Figure 1. Structural composition of EBHAs.

In the normal working mode, EBHAs receives the command signal of the controller, collects the feedback signal of the LVDT installed in the hydraulic cylinder, controls the electro hydraulic servo valve to distribute the oil after comparison and calculation, drives the piston to extend or retract, and completes the servo loop control. The control block diagram is shown in Figure 2. In this working mode, the performance of the electro hydraulic servo valve directly determines the performance of the normal working mode of the system, so the servo performance of the electro hydraulic servo valve is very important. Because the electro hydraulic servo valve itself has pressure feedback, only the conventional position closed loop control mode is used here.

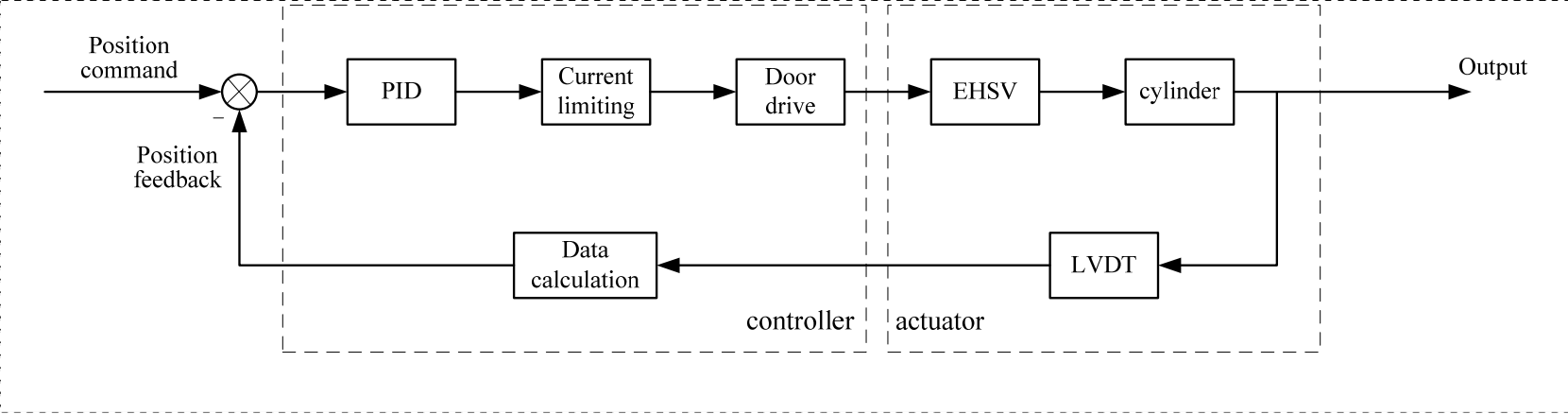

Figure 2. Control schematic diagram of normal working mode.

In the backup working mode, the EBHAs receives the command signal of the controller, collects the motor current and speed signal in the actuator and the feedback signal of the LVDT installed in the hydraulic cylinder, drives the hydraulic pump to move through the control motor after comparison and calculation, distributes the oil to the two chambers of the hydraulic cylinder, so as to drive the piston to extend or retract, and completes the servo loop control. Its control schematic diagram is shown in Figure 3. In this working mode, the servo performance of the motor pump directly determines the performance of the backup working mode of the system, so the servo performance of the motor pump is very important. The double closed-loop control mode of "current loop + speed loop" is adopted to realize the precise control of motor pump.

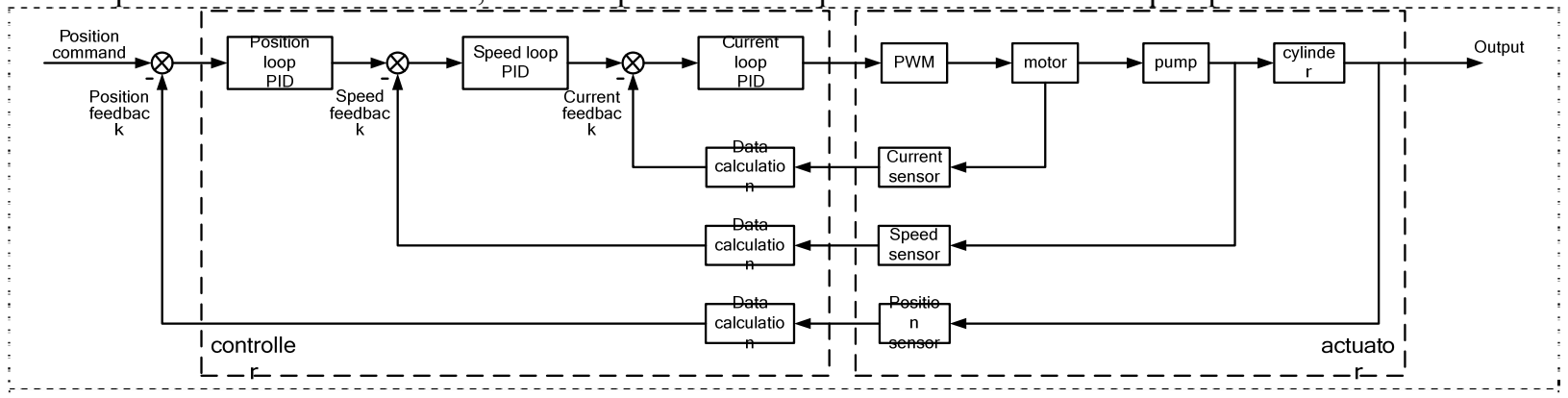

Figure 3. Control schematic diagram of backup working mode. 


\section{EBHAs mathematical model}

There are many components in the EBHAs. This paper only introduces the mathematical model of the main components such as controller, electro-hydraulic servo valve, motor driver, motor, hydraulic pump, actuator and pipeline.

\subsection{Mathematical model of controller}

In the normal working mode, the control mode of position closed loop is adopted, and in the backup working mode, the control mode of "position loop + speed loop + current loop" is adopted, all of which adopt the digital PID control law. The mathematical model can be described as follows.

$$
D(z)=K_{p}+K_{i} \frac{T z}{z-1}+K_{d} \frac{z-1}{T z}
$$

where, $K_{p}$ is the adjustable proportional coefficient of the digital PID, $K_{i}=K_{p} T / T_{i}, K_{d}=K_{p} \tau / T, T$ is the sampling period, $T_{i}$ is the adjustable integral time constant of digital PID, $\tau$ is the adjustable differential time constant of digital PID.

\subsection{Mathematical model of motor driver}

In the backup working mode, the motor adopts SVPWM control method. In order to facilitate the solution, the rotation coordinate system is transformed into the orientation coordinate system through the coordinate transformation (Clark transformation and park transformation) to realize the adjustment of each physical quantity. The structure of space vector control is shown in Figure 4.

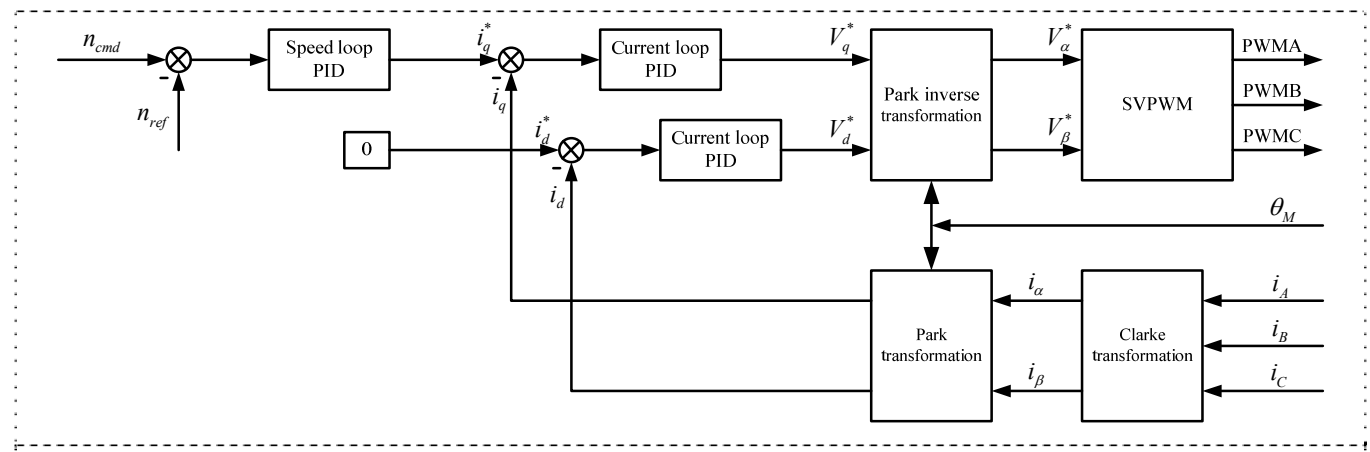

Figure 4. Structure of space vector control.

\subsection{Mathematical model of motor}

Rotor voltage balance equation can be described as follow.

$$
U_{a}=R_{a} i_{a}+L_{a} \frac{d i_{a}}{d t}+E_{a}
$$

where, $U_{a}$ is the armature voltage ( V ), $E_{a}$ is the induced potential ( V ), $R_{a}$ is the total resistance of the armature circuit $(\Omega), L_{a}$ is the armature inductance $(\mathrm{H}), i_{a}$ is the armature current (A ).

Rotor induced potential equation can be described as follow.

$$
E_{a}=K_{e} \Omega_{a}
$$

where, $K_{e}$ is the potential constant $(\mathrm{Vs} / \mathrm{rad}), \Omega_{a}$ is the rotor speed ( $\mathrm{rad} / \mathrm{s})$.

Torque balance equation can be described as follow.

$$
T_{e}-T_{L}=J_{m} \frac{d \Omega_{a}}{d t}+B_{m} \Omega_{a}
$$

where, $T_{e}$ is the electromagnetic torque $(\mathrm{Nm}), T_{L}$ is the total load torque ( $\mathrm{Nm}), T_{L}=T_{0}+T_{f}, T_{0}$ is the no-load resistance torque $(\mathrm{Nm}), T_{f}$ is the load torque $(\mathrm{Nm}), J_{m}$ is the moment of inertia $\left(\mathrm{kgm}^{2}\right), B_{m}$ is the damping coefficient.

\subsection{Mathematical model of hydraulic pump}

Suppose that the two load ports of the hydraulic pump are port $\mathrm{A}$ and port $\mathrm{B}$ respectively. When the motor rotates counter clockwise, port A of the hydraulic pump is the oil drain port and port $\mathrm{B}$ is the oil suction port; when the motor rotates clockwise, port $A$ of the hydraulic pump is the oil suction port and port B is the oil drain port, as shown in Figure 5.
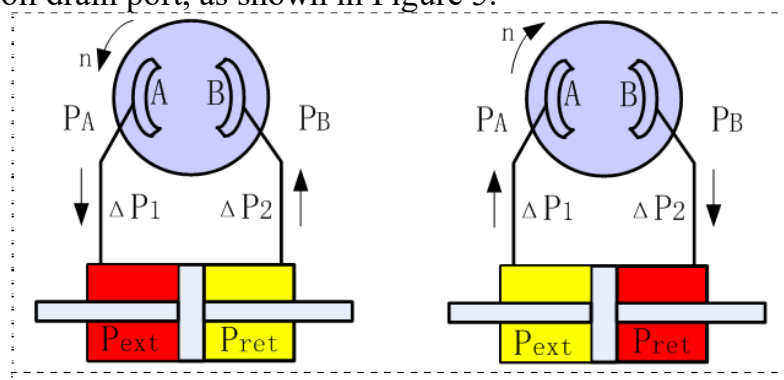

Figure 5. Working principle of hydraulic pump.

Theoretical flow equation of hydraulic pump can be described as follow.

$$
Q_{\text {pump }}=D_{p} n
$$

where, $D_{p}$ is the displacement of the hydraulic pump $(\mathrm{mL} / \mathrm{r}) ; n$ is the speed of the motor $(\mathrm{r} / \mathrm{min})$.

Pressure calculation formula of hydraulic pump port can be described as follow. 


$$
\begin{aligned}
& P_{A}=P_{e x t}+\Delta P_{1}-P_{c d}=P_{e x t}+0.5 P_{l o s t}-P_{c d} \\
& P_{B}=P_{r e t}-\Delta P_{2}-P_{c d}=P_{r e t}-0.5 P_{\text {lost }}-P_{c d} \\
& \Delta P_{\text {pump }}=P_{\text {ext }}-P_{r e t}+P_{\text {lost }}
\end{aligned}
$$

where, $P_{A}$ is the pressure of the hydraulic pump at port A, $P_{B}$ is the pressure of the hydraulic pump at port $\mathrm{B}, \Delta P_{\text {pump }}$ is the pressure difference of the hydraulic pump, $P_{e x t}$ is the pressure in the extension chamber of the hydraulic cylinder, $P_{r e t}$ is the pressure in the retraction chamber of the hydraulic cylinder, $P_{\text {lost }}$ is the pressure loss of the pipeline, $P_{c d}$ is the pressure of the accumulator, $\Delta P_{1}$ is the pressure loss along the pipeline corresponding to port $\mathrm{A}$, $\Delta P_{2}$ is the pressure loss along the pipeline corresponding to port $\mathrm{B}$.

Assuming that the flow out of the hydraulic pump is positive and the flow in the hydraulic pump is negative, the flow equation of the hydraulic pump after considering the leakage is as follow.

$$
\begin{aligned}
& Q_{\text {pump } A}=D_{p} n-K_{p n} \Delta P_{p u m p}-K_{p w} P_{A} \\
& Q_{\text {pump } B}=-D_{p} n+K_{p n} \Delta P_{p u m p}-K_{p w} P_{B}
\end{aligned}
$$

where, $Q_{\text {pumpA }}$ is the flow of the hydraulic pump at port A, $Q_{\text {pumpB }}$ is the flow of the hydraulic pump at port B, $K_{p n}$ is the internal leakage coefficient of the hydraulic pump, $K_{p w}$ is the external leakage coefficient of the hydraulic pump.

There is mechanical friction loss in the working process of the hydraulic pump. For the axial piston pump, the mechanical friction loss mainly occurs between the bottom of the cylinder body and the working surface of the oil distribution plate, the shaft and the bearing, the slipper and the swash plate, the piston and the piston cavity, the piston ball head and the slipper ball socket. The mechanical efficiency of axial piston pump is generally about $0.85 \sim 0.9$. The torque equation of the hydraulic pump is can be described as follow.

$$
D_{p} \times \Delta P_{\text {pump }}+T_{\text {torque_Loss_Sum }}=T_{\text {pump_torq }}
$$

where, $T_{\text {pump_torq }}$ is the input torque of the hydraulic pump, $T_{\text {torque_Loss_Sum }}$ is the mechanical friction loss of hydraulic pump.

\subsection{Mathematical model of electro hydraulic servo valve}

Suppose that the two load ports of the electro hydraulic servo valve are port A and port B respectively. When the current is greater than zero, the extension chamber of the hydraulic cylinder communicates with the high-pressure oil; when the current is less than zero, the retraction chamber of the hydraulic cylinder communicates with the high-pressure oil, as shown in Figure 6.

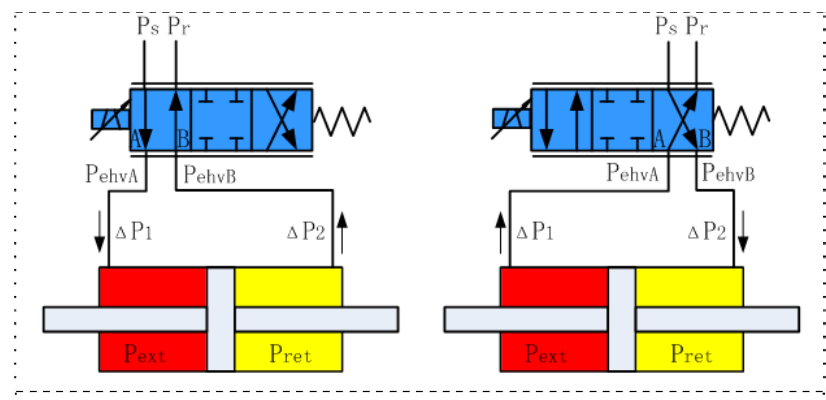

Figure 6. Working principle of electro hydraulic servo valve.

The relationship between the displacement of the valve core and the current of the electro hydraulic servo valve can be described by the second-order transfer function.

$$
G_{s v}(s)=\frac{x_{v}(s)}{i(s)}=\frac{K_{s v}}{\frac{s^{2}}{\omega_{s v}{ }^{2}}+\frac{2 \varsigma_{s v}}{\omega_{s v}} s+1}
$$

where, $x_{v}(s)$ is the Laplace transform of valve core displacement, $i(s)$ is the Laplace transform of valve current, $K_{s v}$ is the gain of valve core displacement, $\omega_{s v}$ is the undamped natural frequency of valve core displacement, and $\varsigma_{s v}$ is the damping ratio of valve core displacement.

Pressure calculation formula of electro hydraulic servo valve port can be described as follow.

$$
\begin{gathered}
P_{e h v A}=P_{e x t}+\Delta P_{1}-P_{c d}=P_{e x t}+0.5 P_{\text {lost }}-P_{c d} \\
P_{e h v B}=P_{r e t}-\Delta P_{2}-P_{c d}=P_{r e t}-0.5 P_{\text {lost }}-P_{c d} \\
\Delta P_{e h v}=P_{e x t}-P_{r e t}+P_{\text {lost }}
\end{gathered}
$$

where, $P_{\text {ehvA }}$ is the pressure of the electro hydraulic servo valve at port $\mathrm{A}, P_{\text {ehvB }}$ is the pressure of the electro hydraulic servo valve at port $\mathrm{B}$, and $\Delta P_{e h v}$ is the pressure difference of the electro hydraulic servo valve.

According to the flow formula, the theoretical flow of the electro hydraulic servo valve can be described as follow.

$$
\begin{aligned}
Q_{e h v 0 A} & =C_{d} x_{v} w \sqrt{\frac{2\left[P_{s}-\left(P_{e x t}+0.5 P_{\text {lost }}-P_{c d}\right)\right]}{\rho}} \\
Q_{e h v 0 B} & =C_{d} x_{v} w \sqrt{\frac{2\left[\left(P_{r e t}-0.5 P_{\text {lost }}-P_{c d}\right)-P_{r}\right]}{\rho}}
\end{aligned}
$$

Where, $x_{v}$ is the displacement of the valve core of the electro hydraulic servo valve, $w$ is the port area gradient of the electro hydraulic servo valve, $P_{s}$ is the rated oil supply pressure of the system, and $P_{r}$ is the oil return pressure of the system.

Assuming that the flow out of the electro hydraulic servo valve is positive and the flow in the electro hydraulic servo valve is negative, the flow equation of the electro hydraulic servo valve after considering the leakage is as follows.

$$
\begin{aligned}
& Q_{e h v A}=Q_{e h v 0 A}-K_{e n} \Delta P_{e h v}-K_{e w} P_{e h v A} \\
& Q_{e h v B}=-Q_{e h v 0 B}+K_{e n} \Delta P_{e h v}-K_{e w} P_{e h v B}
\end{aligned}
$$

where, $Q_{\text {ehvA }}$ is the flow of the electro hydraulic servo valve at port A, $Q_{e h v B}$ is the flow of the electro hydraulic 
servo valve at port $\mathrm{B}, K_{e n}$ is the internal leakage coefficient of the electro hydraulic servo valve, and $K_{e w}$ is the external leakage coefficient of the electro hydraulic servo valve.

\subsection{Mathematical model of actuator}

There are two working modes for the movement of the hydraulic cylinder, oil inlet in the left chamber, oil return in the right chamber (the piston rod extends); oil return in the left chamber and oil inlet in the right chamber (the piston rod retracts). The schematic diagram of actuator movement is shown in Figure 7.
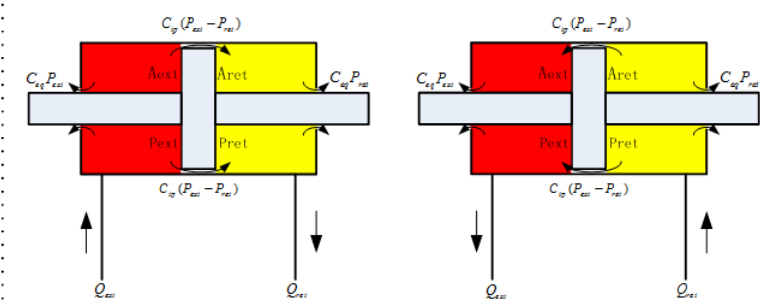

Figure 7. Schematic diagram of actuator movement.

The flow continuity equation of the hydraulic cylinder extension chamber can be described as follow.

$$
Q_{\text {ext }}=A_{e x t} \frac{d\left(X_{p}-X_{c}\right)}{d t}+C_{i p}\left(P_{e x t}-P_{r e t}\right)+C_{e q} P_{e x t}+Q_{\text {leak } 1}+\frac{V_{e x t}}{\beta_{e}} \frac{d P_{e x t}}{d t}
$$

The flow continuity equation of the hydraulic cylinder retraction chamber can be described as follow.

$$
Q_{r e t}=-A_{r e t} \frac{d\left(X_{p}-X_{c}\right)}{d t}-C_{i p}\left(P_{e x t}-P_{r e t}\right)+C_{e q} P_{r e t}+Q_{\text {leak } 2}+\frac{V_{r e t}}{\beta_{e}} \frac{d P_{r e t}}{d t}
$$

where, $C_{i p}$ is the internal leakage coefficient, $C_{e q}$ is the external leakage coefficient, $\beta_{e}$ is the effective bulk modulus of elasticity, $X_{p}$ is the displacement of the piston, $X_{c}$ is the displacement of the cylinder, $A_{e x t}$ is the effective area of the hydraulic cylinder extension chamber, $A_{\text {ret }}$ is the effective area of the hydraulic

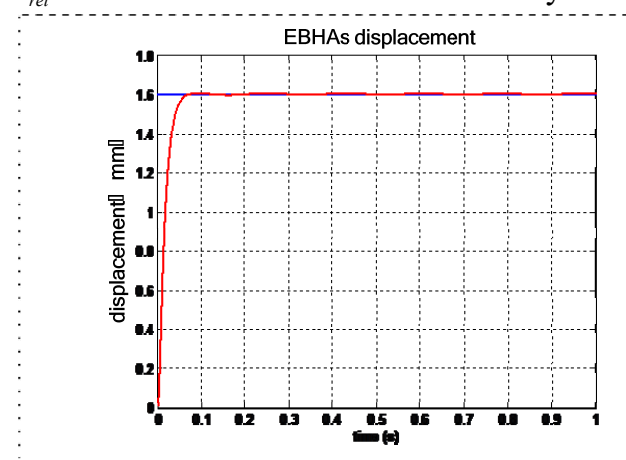

cylinder retraction chamber, $Q_{\text {leak } 1}$ is the leakage flow of the pipeline connected with the hydraulic cylinder extension chamber, $Q_{\text {leak } 2}$ is the leakage flow of the pipeline connected with the hydraulic cylinder retraction chamber, $V_{e x t}$ is the volume of the hydraulic cylinder extension chamber, $V_{e x t}=V_{e x t 0}+A_{e x t}\left(X_{p}-X_{c}\right), V_{r e t}$ is the volume of the hydraulic cylinder retraction chamber, $V_{\text {ret }}=V_{\text {ret } 0}-A_{\text {ret }}\left(X_{p}-X_{c}\right), V_{e x t 0}$ is the initial volume of the hydraulic cylinder extension chamber, $V_{\text {ret }}$ is the initial volume of the hydraulic cylinder retraction chamber.

\section{EBHAs simulation and result analysis}

The simulation model of EBHAs is established in Matlab / Simulink environment, as shown in Figure 8.

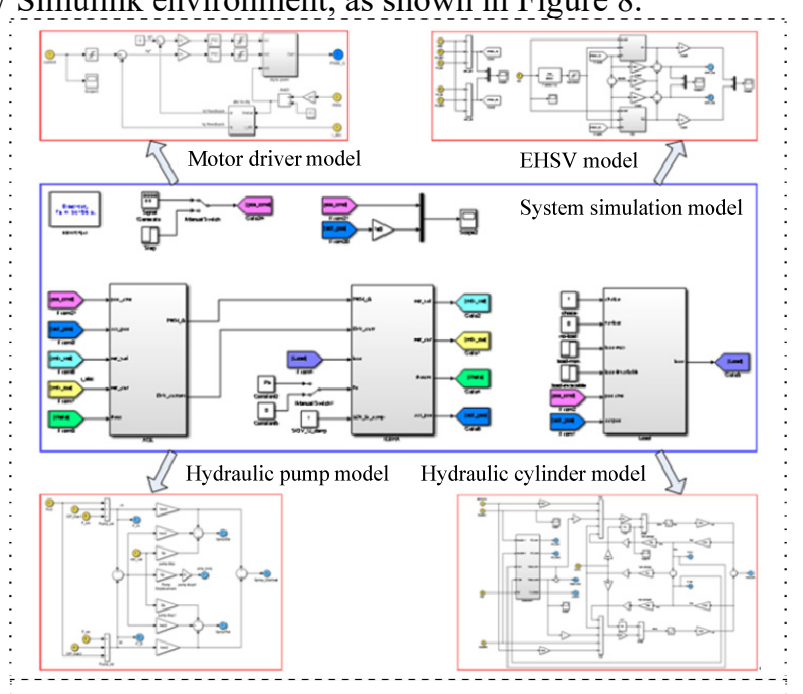

Figure 8. Simulation model of EBHAs.

In the normal working mode, input step command with an amplitude of $1.6 \mathrm{~mm}$ or sine command with an amplitude of $1.6 \mathrm{~mm}$ and frequency of $8 \mathrm{~Hz}$, in order to observe the response characteristics of the system.

Figure 9. Simulation results of normal working mode of EBHAs.

In the backup working mode, input step command with an amplitude of $1.6 \mathrm{~mm}$ or sine command with an amplitude of $1.6 \mathrm{~mm}$ and frequency of $4 \mathrm{~Hz}$, to observe the response characteristics of the system.
The simulation results show that: In the normal working mode, when the step signal with an amplitude of $1.6 \mathrm{~mm}$ is input on no load condition, the response time of the system is less than $50 \mathrm{~ms}$, there is no overshoot, and it can reach a stable state soon; when the sine signal 
with an amplitude of $1.6 \mathrm{~mm}$ and the frequency of $8 \mathrm{~Hz}$ is input, the amplitude attenuation does not exceed $-3 \mathrm{~dB}$, and the phase lag is less than $90^{\circ}$. In the backup working mode, when the step signal with an amplitude of $1.6 \mathrm{~mm}$ is input on no load condition, the response time of the system is less than $150 \mathrm{~ms}$, there is no overshoot, and it can reach a stable state soon; when the sine signal with an amplitude of $1.6 \mathrm{~mm}$ and the frequency of $4 \mathrm{~Hz}$ is input,

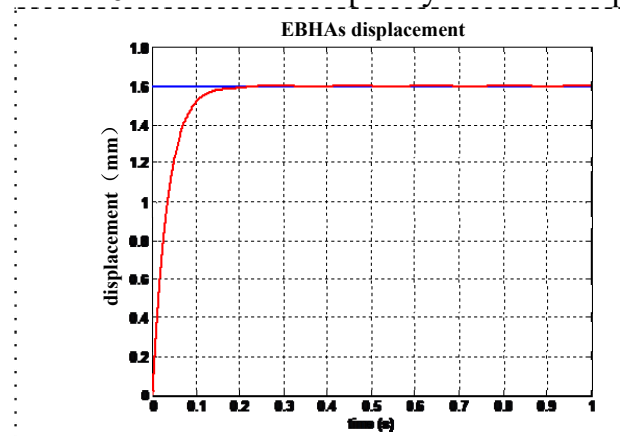

Figure 10. Simulation results of backup working mode of EBHAs.

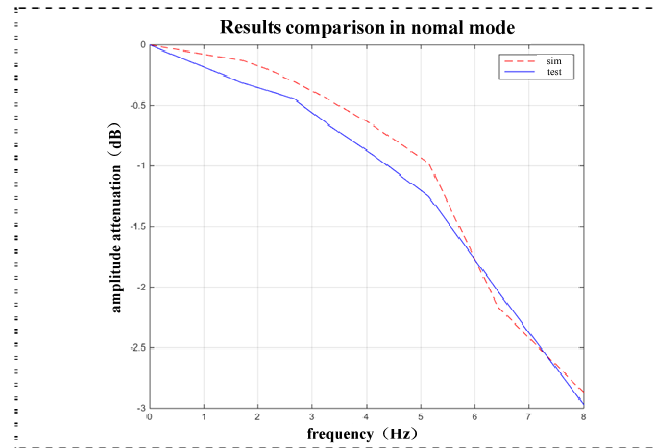

Figure 11. Comparison between simulation results and test results in the normal mode of EBHAs.

Figure 11 shows that, when given a sine signal with an amplitude of $1.6 \mathrm{~mm}$ and frequency of $0 \sim 8 \mathrm{~Hz}$, the simulation results of EBHAs are very approach to the test results in the normal working mode. The maximum error of amplitude attenuation is not more than $9.2 \%$, the maximum error of phase lag is not more than $3.5 \%$, and the performance error rate of the whole model is not more than $10 \%$.

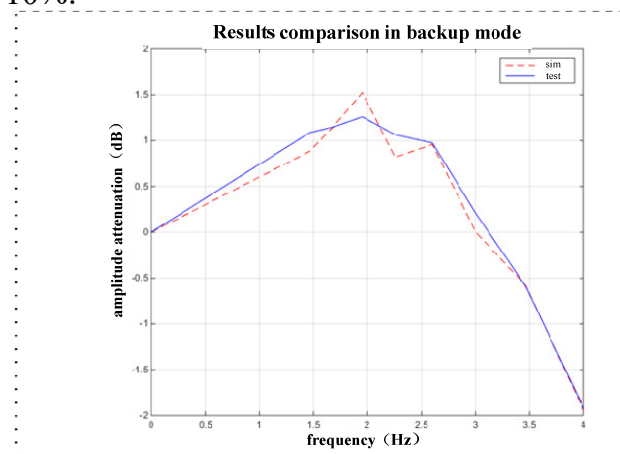

Figure 12. Comparison between simulation results and test results in the backup mode of EBHAs.

Figure 12 shows that, when given a sine signal with an amplitude of $1.6 \mathrm{~mm}$ and frequency of $0 \sim 4 \mathrm{~Hz}$, the simulation results of EBHAs are very approach to the test results in the backup working mode. The maximum the amplitude attenuation does not exceed $-3 \mathrm{~dB}$, and the phase lag is less than $90^{\circ}$.

In the normal working mode, the performance sweep simulation test is carried out for EBHAs. Input a sine command with an amplitude of $1.6 \mathrm{~mm}$ and frequency of $0 \sim 8 \mathrm{~Hz}$, the comparison between the simulation results and the test results is shown in Figure 11.
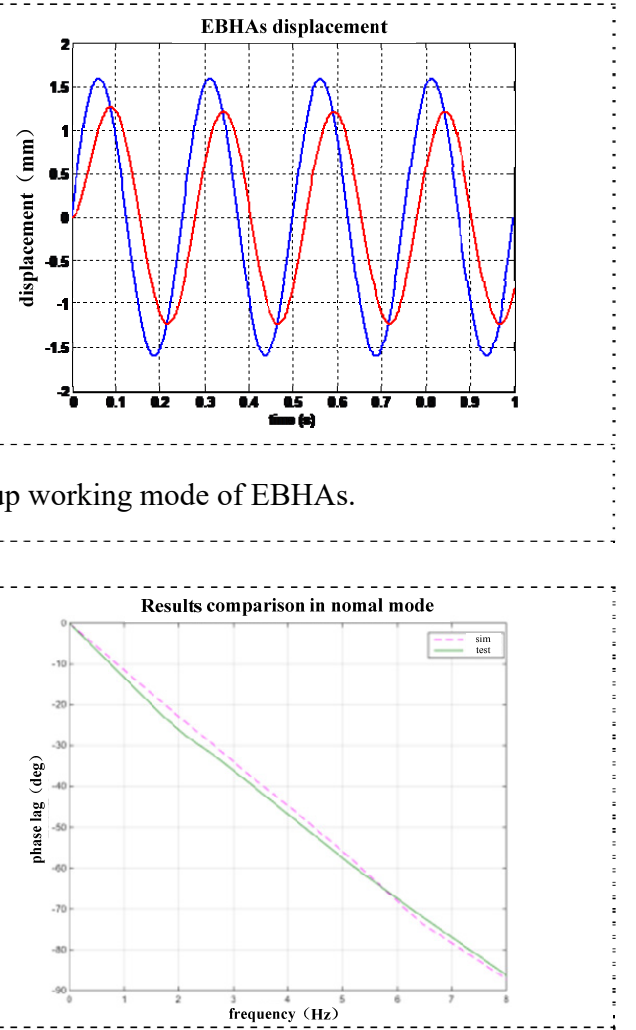

In the backup working mode, the performance sweep simulation test is carried out for EBHAs. Input a sine command with an amplitude of $1.6 \mathrm{~mm}$ and frequency of $0 \sim 4 \mathrm{~Hz}$, the comparison between the simulation results and the test results is shown in Figure 12.

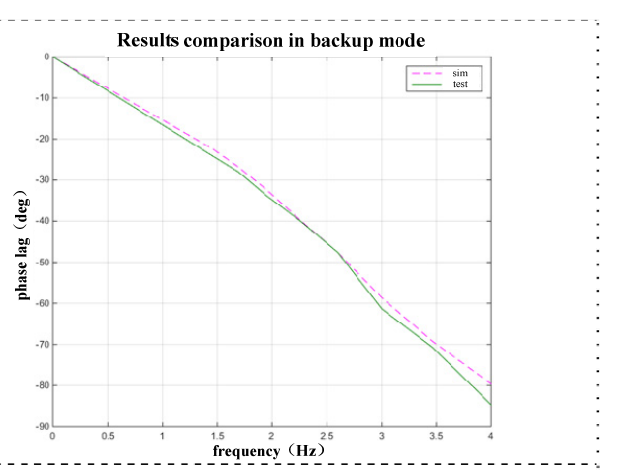

error of amplitude attenuation is not more than $8.3 \%$, the maximum error of phase lag is not more than $5.6 \%$, and the performance error rate of the whole model is not more than $10 \%$. 


\section{Conclusion}

Taking the EBHAs as an example, the mathematical model and simulation model of EBHAs system are established. The performance in the normal and backup modes are simulated under different frequencies, and compared with the corresponding test results.

The results of simulation and test show that: (1) when given the step signal with an amplitude of $1.6 \mathrm{~mm}$, the system has no overshoot in the normal or backup working modes, and can reach a stable state soon, meeting the requirements of system rapidity and stability. (2) when given a sine signal with an amplitude of $1.6 \mathrm{~mm}$, the bandwidth of system is $8 \mathrm{~Hz}$ in the normal working mode, and $4 \mathrm{~Hz}$ in the backup working mode, meeting the system bandwidth requirements; (3) when given a sine signal with an amplitude of $1.6 \mathrm{~mm}$, and frequency of $0 \sim 8 \mathrm{~Hz}$ in the normal working mode and $0 \sim 4 \mathrm{~Hz}$ in the backup working mode, the system simulation results are consistent with the test results, and the error is less than $10 \%$, which provides an effective way for the performance simulation of similar products in the early stage of development.

\section{References}

1. Li Jun, Fu Yongling, Wang Zhanlin. Present development status and key technology research of airborne electro-hydrostatic actuation system [J]. Aeronautical Manufacturing Technology, 2005, 11:73-77.

2. Ma Jiming, Fu Yongling, Li Jun, Gao Bo. Design, simulation and analysis of integrated electrical hydrostatic actuator [J]. Acta Aeronautica Et Astronautica Sinica, 2005, 26(1):79-83.

3. Kang Rongjie, Jiao Zongxia, Jean Charles Mare, Shang Yaoxing, Wu Shuai. Nonlinear block diagram model and robust control of electro-hydrostatic actuator [J]. Acta Aeronautica Et Astronautica Sinica, 2009,30(3):518-525.

4. Li Weiyi, Cheng Yang. System design and simulation of electro-hydrostatic actuator [J]. Measurement \& Control Technology, 2010, 29(11):59-62.

5. Li Yongfeng. Electrically powered actuation system design for long range wide body commercial aircraft [J]. Acta Aeronautica Et Astronautica Sinica, 2017, 38(S1):721531.

6. Xia Liqun, Xie Zengrong. Research on civil aircraft actuator [C]. High Level Forum on Key Technologies of Large aircraft and 2007 academic conference of CAAC, Shenzhen, 2007.

7. Chen Cai. Discussion on EHA/EBHA/EMA technology of civil aircraft [J]. Science \& Technology Vision, 2017, 04:319. 